MATHEMATICS OF COMPUTATION

Volume 72 , Number 242 , Pages 901-911

S 0025-5718(02)01442-4

Article electronically published on June 25, 2002

\title{
ON THE LINEAR INDEPENDENCE MEASURE OF LOGARITHMS OF RATIONAL NUMBERS
}

\author{
QIANG WU
}

\begin{abstract}
In this paper we give a general theorem on the linear independence measure of logarithms of rational numbers and, in particular, the linear independence measure of $1, \log 2, \log 3, \log 5$ and of $1, \log 2, \log 3, \log 5, \log 7$. We also give a method to search for polynomials of smallest norm on a real interval $[a, b]$ which may be suitable for computing or improving the linear independence measure of logarithms of rational numbers.
\end{abstract}

\section{INTRODUCTION}

For an irrational real number $\alpha$, the real number $\mu>0$ is said to be an irrationality measure of $\alpha$ if, for any $\varepsilon>0$, there exists $q_{0}=q_{0}(\varepsilon)>0$, such that

$$
|\alpha-p / q| \geq q^{-\mu-\varepsilon}
$$

for all integers $p$ and $q$ with $q>q_{0}$. If $q_{0}(\varepsilon)$ is effectively computable, we say that $\mu$ is an effective irrationality measure of $\alpha$. The minimum of these numbers $\mu$ is denoted by $(\mu) \alpha$.

In the same way if $\alpha_{0}, \alpha_{1}, \ldots, \alpha_{n}$ are real numbers linearly independent over $\mathbb{Q}$, we say that $\nu>0$ is a linear independence measure of $\alpha_{0}, \alpha_{1}, \ldots, \alpha_{n}$ if for any $\varepsilon>0$ there exists $H_{0}=H_{0}(\varepsilon)>0$ such that

$$
\left|p \alpha_{0}+q_{1} \alpha_{1}+\cdots+q_{n} \alpha_{n}\right| \geq H^{-\nu-\varepsilon}
$$

for all integers $p, q_{1}, \ldots, q_{n}$, with $H=\max \left\{\left|q_{1}\right|, \ldots,\left|q_{n}\right|\right\} \geq H_{0}$.

The minimum of these numbers $\nu$ is denoted by $\nu\left(\alpha_{0}, \alpha_{1}, \ldots, \alpha_{n}\right)$. We remark that, with this notation, we have $\mu(\alpha)=\nu(1, \alpha)+1$. We are interested here in the linear independence (or irrationality) measure of logarithms of rational numbers. Baker [?] gave effective lower bounds of nonvanishing linear forms of logarithms

$$
\Lambda=\beta_{0}+\beta_{1} \log \alpha_{1}+\cdots+\beta_{n} \log \alpha_{n},
$$

where $\alpha_{i}$ and $\beta_{i}$ are algebraic numbers. In the particular case where $\alpha_{i}$ are rationals and $\beta_{i}$ integers, we obtain linear independence measures (see M. Waldschmidt WA] or G. Wüstholz and Baker [BW]). However, the linear independence (or irrationality) measures are very large; for example we have $\mu(\log 2) \leq 10^{22}$.

In the case of one logarithm, the best irrationality measures are obtained by Siegel's method. For example, the value $\mu(\log 2)<3.891399$ was obtained by Rukhadze [RU]. For a more detailed exposition we refer the reader to the paper by Viola VI.

Received by the editor April 17, 2001 and, in revised form, September 5, 2001.

2000 Mathematics Subject Classification. Primary 11J82, 11J86.

(C)2002 American Mathematical Society 
Results on linear independence of logarithms of rational numbers were given by Nikisin [NI and Danilov [DA] using Padé approximants. In the case of the linear independence of $1, \log r_{1}, \log r_{2}$, where $r_{1}$ and $r_{2}$ are rationals, Rhin [RH] showed that the results of Nikisin and Danilov could be improved. In particular he obtained a linear independence measure of $1, \log 2, \log 3$ less than 7.616 by considering the two integrals

$$
\int_{2}^{3} \frac{H_{n}(x)}{x^{n}} \frac{d x}{n} \text { and } \int_{3}^{4} \frac{H_{n}(x)}{x^{n}} \frac{d x}{x}
$$

where the polynomial $H_{n}$ is of the type $b_{0} \prod_{i=1}^{6} Q_{i}(x)^{\left[b_{i} n\right]}$ and $b_{0}, b_{1}, \ldots, b_{6}$ are positive constants and the polynomials $Q_{i}$ are $Q_{1}=x-2, Q_{2}=x-3, Q_{3}=$ $x-4, Q_{4}=5 x-12, Q_{5}=17 x^{2}-102 x+144, Q_{6}=19 x^{2}-104 x+144$. This is the best linear independence measure known for $1, \log 2, \log 3$, and it gives the best irrationality measure known of $\log 3$ (8.616). However, Rhin did not give a theoretical justification for the use of the polynomials $Q_{4}, Q_{5}, Q_{6}$. We propose in this paper to give a general method for determining such factors by using the following generalization of the integer transfinite diameter of a real interval.

Let $f$ be a positive function on the real interval $[a, b]$ and $\Delta$ a positive integer. We define

$$
t_{\mathbb{Z},(f, \Delta)}([a, b])=\inf _{p \in I_{n, \Delta}} \max _{x \in[a, b]}\left(|p(x)|^{1 / 2 n} f(x)\right)
$$

where $I_{n, \Delta}$ is the set of polynomials of $\mathbb{Z}[x]$ with degree $2 n$ belonging to the ideal $(x \mathbb{Z}[x]+\delta \mathbb{Z}[x])^{n}$. When $f(x)=1$ and $\Delta=1$ we get the usual integer transfinite diameter.

We show that the polynomials $Q_{1}$ to $Q_{6}$ in (1.4) are obtained as factors of polynomials $P$ of $I_{n, \Delta}$ having a small degree and a minimal norm. We will use a generalization of the Müntz-Legendre polynomials to calculate these polynomials with a small degree (see also Amoroso [AM1, AM2], Borwein and Erdelyi [BE], or Flammang, Rhin and Smyth [FRS] ).

In Section 2 we give a general theorem on the linear independence measure of logarithms of rational numbers. In Section 3, we prove for $H=\max _{i}\left|q_{i}\right|$ sufficiently large

$$
\begin{gathered}
\left|p+q_{1} \log 2+q_{2} \log 3+q_{3} \log 5\right| \geq H^{-15.27049-\varepsilon}, \\
\left|p+q_{1} \log 2+q_{2} \log 3+q_{3} \log 5+q_{4} \log 7\right| \geq H^{-256.865-\varepsilon}
\end{gathered}
$$

and give other improvements of the linear independence measure of logarithms of rational numbers given by Rhin and Toffin $[\mathrm{RT}]$. In Section 4 we generalize the Müntz-Legendre polynomials and deduce an inequality on the coefficients of the polynomials of small norm on a real interval $[a, b]$ and we explain how to use algorithm LLL [LLL and the preceding inequality to calculate for small degrees the polynomial of $I_{n, \Delta}$ of smallest norm. In Section 5 we explain how to seek polynomials which may be used to improve the linear independence measure of logarithms of rational numbers. 


\section{A GENERAL THEOREM ON THE LINEAR INDEPENDENCE MEASURE OF LOGARITHMS OF RATIONAL NUMBERS}

We first prove the following lemma.

Lemma 1. Let $m \in \mathbb{N}^{*}$ and $\gamma_{1}, \ldots, \gamma_{m}$ be $m$ real numbers. Suppose that for any $n \geq 1$ there exist integers $r_{n}>0, p_{n}^{(1)}, \ldots, p_{n}^{(m)}$ such that if $\varepsilon_{n}^{(i)}=r_{n} \gamma_{i}-p_{n}^{(i)}$ $(1 \leq i \leq m)$, then $\varepsilon_{n}^{(i)} \neq 0$ for $1 \leq i \leq m$ and

$$
\lim _{n \rightarrow \infty} \frac{1}{n} \log \left(\left|r_{n}\right|\right) \leq \sigma, \quad \lim _{n \rightarrow \infty} \frac{1}{n} \log \left(\left|\varepsilon_{n}^{(i)}\right|\right)=-\tau^{(i)}, \quad 1 \leq i \leq m,
$$

where $\sigma, \tau^{(i)}(1 \leq i \leq m)$ are positive numbers. Let $\tau=\min _{1 \leq i \leq m}\left(\tau^{(i)}\right)$.

If for any $i \neq j, \tau^{(i)} \neq \tau^{(j)}$, then $1, \gamma_{1}, \ldots, \gamma_{m}$ are linearly independent over $\mathbb{Q}$ and, for any $\varepsilon>0$, there exists a positive integer $H_{0}(\varepsilon)$ such that

$$
\left|p+q_{1} \gamma_{1}+\cdots+q_{m} \gamma_{m}\right| \geq H^{\frac{-\sigma}{\tau}-\varepsilon}
$$

for all integers $p, q_{i},(1 \leq i \leq m)$ with $H=\max _{1 \leq i \leq m}\left\{\left|q_{i}\right|\right\} \geq H_{0}(\varepsilon)$.

Proof. We may suppose that $\varepsilon<\tau$. Let $\Lambda_{m}=p+q_{1} \gamma_{1}+q_{2} \gamma_{2}+\cdots+q_{m} \gamma_{m}$ for a fixed integer $m$. We can suppose, if necessary by replacing $m$ by a smaller integer, that $q_{1}, q_{2}, \ldots, q_{m}$ are all nonzero. We have

$$
\Lambda_{m}=p+\sum_{i=1}^{m} q_{i}\left(\frac{p_{n}^{(i)}+\varepsilon_{n}^{(i)}}{r_{n}}\right)=\frac{p r_{n}+\sum_{i=1}^{m} q_{i} p_{n}^{(i)}}{r_{n}}+\frac{\sum_{i=1}^{m} q_{i} \varepsilon_{n}^{(i)}}{r_{n}}=\frac{A_{n}}{r_{n}}+\frac{\omega_{n}}{r_{n}}
$$

where

$$
A_{n}=p r_{n}+\sum_{i=1}^{m} q_{i} p_{n}^{(i)} \quad \text { and } \quad \omega_{n}=\sum_{i=1}^{m} q_{i} \varepsilon_{n}^{(i)}
$$

We prove that $1, \gamma_{1}, \ldots, \gamma_{m}$ are linearly independent over $\mathbb{Q}$ by induction on $m$.

For $m=1$ the result is Lemma 3.1 of Hata [HA].

For $m \geq 2$, suppose that the lemma is true for $m-1$. Then

$$
\Lambda_{m-1}=p+q_{1} \gamma_{1}+\cdots+q_{m-1} \gamma_{m-1} \neq 0 .
$$

a) If there exists an integer $n_{0}$ such that for $n \geq n_{0}, A_{n} \in \mathbb{Z}^{*}$ we have $\left|\frac{A_{n}}{r_{n}}\right| \geq \frac{1}{r_{n}}$. Since for any $\varepsilon>0$ there exists $n_{0}(\varepsilon)$ such that for $n \geq n_{0}(\varepsilon)$, we have $\left|\omega_{n}\right| \leq$ $m H e^{(-\tau+\varepsilon) n}$, then we can choose $n_{1}(\varepsilon)$ such that $n \geq n_{1}(\varepsilon),\left|\omega_{n}\right| \leq \frac{1}{2}$. We have $\left|\Lambda_{m}\right| \geq\left|\frac{A_{n}}{r_{n}}\right|-\left|\frac{\omega_{n}}{r_{n}}\right| \geq \frac{1}{2 r_{n}}$ and then $1, \gamma_{1}, \ldots, \gamma_{m}$ are linearly independent over $\mathbb{Q}$.

b) If $A_{n}=0$ for an infinite number of integers $n$, then $\Lambda_{m}=\frac{\omega_{n}}{r_{n}}$. We have $\Lambda_{m}=0$ because $r_{n}$ is a positive integer and $\omega_{n} \rightarrow 0$ when $n \rightarrow \infty$, so $\omega_{n}=0$, i.e., $\sum_{i=1}^{m} q_{i} \varepsilon_{n}^{(i)}=0$

Let $\tau=\tau^{(l)}=\min _{i}\left(\tau^{(i)}\right)$, so that $\sum_{i \neq l} q_{i} \frac{\varepsilon_{n}^{(i)}}{\varepsilon_{n}^{(l)}}+q_{l}=0$ and, moreover, for all $i$, $\lim _{n \rightarrow \infty} \frac{1}{n} \log \left|\varepsilon_{n}^{(i)}\right|=-\tau^{(i)}$. Thus, we have, for any real number $\theta>0$ and $n \geq n(\theta)$, $e^{\left(-\tau^{(i)}+\theta\right) n} \geq\left|\varepsilon_{n}^{(i)}\right| \geq e^{\left(-\tau^{(i)}-\theta\right) n}$. Hence for all $i \neq l$, there exists an integer $n_{1}$ such that for all $n \geq n_{1},\left|\frac{\varepsilon_{n}^{(i)}}{\varepsilon_{n}^{(l)}}\right| \leq e^{\left(\tau-\tau^{(i)}+2 \theta\right) n}$ with $2 \theta<-\min _{i \neq l}\left(\tau-\tau^{(i)}\right)$, and so $\lim _{n \rightarrow \infty}\left|\frac{\varepsilon_{n}^{(i)}}{\varepsilon_{n}^{(l)}}\right|=0$ for all $i \neq l$, i.e., $\lim _{n \rightarrow \infty}\left(\sum_{i \neq l} q_{i} \frac{\varepsilon_{n}^{(i)}}{\varepsilon_{n}^{(l)}}+q_{l}\right)=q_{l}$ and so $q_{l}=0$. Thus we obtain $\Lambda_{m-1}=\sum_{i \neq l} q_{i} \gamma_{i}+p=0$, i.e., $1, \gamma_{1}, \ldots, \gamma_{m-1}$ are not 
linearly independent over $\mathbb{Q}$. We have a contradiction. Hence, for any integer $m$, if $\tau^{(i)} \neq \tau^{(j)}$ for $i \neq j, 1, \gamma_{1}, \ldots, \gamma_{m}$ are linearly independent over $\mathbb{Q}$.

It is clear, from b), that the set $\Omega=\left\{n \geq 1 ; A_{n} \neq 0\right\}$ is infinite. So for any $\varepsilon>0$, we can define a sufficiently small $\delta \equiv \delta(\varepsilon) \in] 0, \tau / 6[$ satisfying $\frac{\sigma+\delta}{\tau-3 \delta}<\frac{\sigma}{\tau}+\frac{\varepsilon}{2}$. So there is an integer $n(\varepsilon) \geq n_{0}$ such that $e^{(\sigma-\delta) n} \leq\left|r_{n}\right| \leq e^{(\sigma+\delta) n}$ and $\max _{1 \leq i \leq m}\left|\varepsilon_{n}^{(i)}\right| \leq e^{-(\tau-\delta) n}$.

For all $n \geq n(\varepsilon)$, we define

$$
\begin{gathered}
H_{0}(\varepsilon)=\min \left\{N \geq 1 \mid N^{\frac{\varepsilon}{2}} \geq 2 e^{\sigma+\delta}(2 m)^{\frac{\sigma+\delta}{\tau-\delta}} \text { and } N^{\frac{\varepsilon \tau}{4}} \geq\left(2 e^{\sigma+\delta}\right)^{\tau+\sigma} m^{\sigma+\delta}\right\}, \\
N(H)=\min \left\{N>n(\varepsilon) \mid 2 m H<e^{(\tau-\delta) N}\right\}
\end{gathered}
$$

for all $H>H_{0}(\varepsilon)$. Since $\left|\omega_{n}\right| \leq m H e^{-(\tau-\delta) n}$ for all $n>n(\varepsilon)$, we have $\left|\omega_{n}\right| \leq \frac{1}{2}$ for all $n \geq N(H)$; hence

$$
|\Lambda|=\left|\frac{A_{n}+\omega_{n}}{r_{n}}\right| \geq \frac{1-\left|\omega_{n}\right|}{\left|r_{n}\right|}>\frac{1}{2\left|r_{n}\right|} \geq \frac{1}{2} e^{-(\sigma+\delta) n}
$$

for any $n \in \Omega$ with $n \geq N(H)$ (we have $\left|A_{n}\right| \geq 1$ if $A_{n} \neq 0$, because $A_{n} \in \mathbb{Z}$ ).

Now we consider the following two cases: $N(H) \in \Omega$ and $N(H) \notin \Omega$. In case (1), from the definition of $N(H)$, we have $2 m H \geq e^{(\tau-\delta)(N(H)-1)} \Longrightarrow e^{N(H)} \leq$ $e(2 m H)^{\frac{1}{(\tau-\delta)}}$. Now replacing $n$ by $N(H)$ in $(2.2)$, we always have $A_{n} \neq 0$, and so

$$
|\Lambda| \geq \frac{1}{2} e^{-(\sigma+\delta) N(H)} \geq \frac{1}{2} e^{-\sigma-\delta}(2 m)^{\frac{-\sigma-\delta}{\tau-\delta}} H^{\frac{-\sigma-\delta}{\tau-\delta}} \geq\left(H_{0}(\varepsilon)\right)^{\frac{-\varepsilon}{2}} H^{-\frac{\sigma}{\tau}-\frac{\varepsilon}{2}} \geq H^{\frac{-\sigma}{\tau}-\varepsilon} .
$$

In case (2), let $M(H)$ be the smallest integer satisfying $M(H)>N(H)$ and $M(H) \in \Omega$. Since $A_{M(H)-1}=0$, we have

$$
|\Lambda|=\left|\frac{\omega_{M(H)-1}}{r_{M(H)-1}}\right| \leq m H e^{-(\sigma+\tau-2 \delta)(M(H)-1)} \Longrightarrow e^{M(H)} \leq e\left(\frac{m H}{|\Lambda|}\right)^{\frac{1}{(\sigma+\tau-2 \delta)}}
$$

By taking $n=M(H)$ in $(\underline{2.2})$, we obtain

$$
|\Lambda| \geq \frac{1}{2} e^{-(\sigma+\delta) M(H)} \geq \frac{1}{2} e^{-(\sigma+\delta)}\left(\frac{|\Lambda|}{m H}\right)^{\frac{\sigma+\delta}{\sigma+\tau-2 \delta}}
$$

and so

$$
\begin{aligned}
|\Lambda| & \geq\left(\frac{1}{2}\right)^{\frac{\tau+\sigma-2 \delta}{\tau-3 \delta}}\left(e^{-\sigma-\delta}\right)^{\frac{\tau+\sigma-2 \delta}{\tau-3 \delta}} m^{\frac{-\sigma-\delta}{\tau-3 \delta}} H^{\frac{-\sigma-\delta}{\tau-3 \delta}} \\
& \geq 2^{\frac{-\tau-\sigma}{\tau-3 \tau / 6}}\left(e^{\sigma+\delta}\right)^{\frac{-\tau-\delta}{\tau-3 \tau / 6}} m^{\frac{-\sigma-\delta}{\tau-3 \tau / 6}} H^{-\frac{\sigma}{\tau}-\frac{\varepsilon}{2}} \\
& \geq\left(2^{\tau+\sigma}\left(e^{\sigma+\delta}\right)^{\tau+\sigma} m^{\sigma+\delta}\right)^{-\frac{2}{\tau}} H^{-\frac{\sigma}{\tau}-\frac{\varepsilon}{2}} \\
& \geq\left(H_{0}(\varepsilon)\right)^{\frac{-\varepsilon}{2}} H^{-\frac{\sigma}{\tau}-\frac{\varepsilon}{2}} \geq H^{\frac{-\sigma}{\tau}-\varepsilon} .
\end{aligned}
$$

Remark. The conclusion of Lemma 1 remains true if we replace the condition "for all $i \neq j, \tau^{(i)} \neq \tau^{(j)}$ " by " $1, \gamma_{1}, \ldots, \gamma_{m}$ are linearly independent over $\mathbb{Q}$ ".

We have the following general theorem for the linear independence measure of logarithms of rational numbers. 
Theorem 2. Let $1<a_{1}<\cdots<a_{m}$ be $m$ positive integers and $\Delta=$ $\operatorname{gcd}\left(a_{1}, a_{2}, \ldots, a_{m}\right)$. For any $n \geq 0$, let $H_{n}$ be a polynomial with integer coeffcients, $H_{n} \in(\Delta, x)^{n} \mathbb{Z}[x]$. For any $a_{i}(1 \leq i<m)$ we define

$$
I_{n}\left(a_{i}, a_{i+1}\right)=\int_{a_{i}}^{a_{i+1}} \frac{H_{n}(x)}{x^{n}} \frac{d x}{x} .
$$

Then define $D_{n}=\operatorname{gcd}\left(1,2, \ldots, \max \left(n, \operatorname{deg} H_{n}-n\right)\right)$, and $\lim _{n \rightarrow \infty} \frac{1}{n} \log D_{n}=K$, then we have

$$
D_{n} I_{n}\left(a_{i}, a_{i+1}\right) \in \mathbb{Z}+\mathbb{Z} \log \left(\frac{a_{i+1}}{a_{i}}\right) .
$$

Suppose that there exist distinct positive integers $\tau^{(i)}(1 \leq i<m)$ such that

$$
-\tau^{(i)}=\lim _{n \rightarrow \infty} \frac{1}{n} \log \left(\max _{a_{i} \leq x \leq a_{i+1}} \frac{\left|H_{n}(x)\right|}{x^{n}}\right),
$$

and that there exist real numbers $\rho>0$ and $\tau^{(0)}>0$ such that

$$
\tau^{(0)}=\lim _{n \rightarrow \infty} \frac{1}{n} \log \left(\max _{|z|=\rho}\left|\frac{H_{n}(z)}{z^{n}}\right|\right) .
$$

If $\tau=\min _{1 \leq i \leq m-1}\left(\tau^{(i)}\right)>K$, then for any $\varepsilon>0$, there exist a positive integer $H_{0}(\varepsilon)$ such that

$$
\left|p+q_{1} \log \left(\frac{a_{2}}{a_{1}}\right)+\cdots+q_{i} \log \left(\frac{a_{i+1}}{a_{i}}\right)+\cdots+q_{m-1} \log \left(\frac{a_{m}}{a_{m-1}}\right)\right| \geq H^{-\mu-\varepsilon}
$$

for any integers $p$ and $q_{i}(1 \leq i \leq m-1)$ with $H=\max _{i}\left\{\left|q_{i}\right|\right\} \geq H_{0}(\varepsilon)$, where $H_{0}(\varepsilon)$ is effectively computable and $\mu$ is a positive real number given by

$$
\mu=\frac{\tau^{(0)}+K}{\tau-K} .
$$

Proof. We put $H_{n}(x)=\sum_{j=0}^{\operatorname{deg} H_{n}} B_{j} x^{j}$ and

$$
\begin{aligned}
I_{n} & =\int_{a_{i}}^{a_{i+1}} \frac{H_{n}(x)}{x^{n}} \frac{d x}{x}=\sum_{j=0}^{\operatorname{deg} H_{n}} B_{j} \int_{a_{i}}^{a_{i+1}} x^{j-n-1} d x \\
& =B_{n} \log \left(\frac{a_{i+1}}{a_{i}}\right)+\sum_{j=0}^{n-1} \frac{B_{j}}{j-n}\left(a_{i+1}^{j-n}-a_{i}^{j-n}\right)+\sum_{j=n+1}^{\operatorname{deg} H_{n}} \frac{B_{j}}{j-n}\left(a_{i+1}^{j-n}-a_{i}^{j-n}\right) .
\end{aligned}
$$

Thus, we have, for $j \neq n, B_{j}\left(a_{i+1}^{j-n}-a_{i}^{j-n}\right) \in \mathbb{Z}$ because $H_{n}(x) \in(\Delta, x)^{n} \mathbb{Z}[x]$ and $\Delta=\operatorname{gcd}\left(a_{1}, a_{2}, \ldots, a_{m}\right)$. We then have

$$
\begin{aligned}
D_{n} I_{n}\left(a_{i}, a_{i+1}\right) & =D_{n} B_{n} \log \left(\frac{a_{i+1}}{a_{i}}\right)+D_{n}\left(\sum_{j \neq n} \frac{B_{j}}{j-n}\left(a_{i+1}^{j-n}-a_{i}^{j-n}\right)\right) \\
& \in \mathbb{Z} \log \left(\frac{a_{i+1}}{a_{i}}\right)+\mathbb{Z} .
\end{aligned}
$$

Hence, we have the relations

$$
D_{n} I_{n}\left(a_{i}, a_{i+1}\right)=p_{n, i}+D_{n} B_{n} \log \left(\frac{a_{i+1}}{a_{i}}\right), \quad 1 \leq i \leq m-1,
$$


with $p_{n, i} \in \mathbb{Z}$. Put $D_{n} I_{n}\left(a_{i}, a_{i+1}\right)=\varepsilon_{n}^{(i)}, D_{n} B_{n}=r_{n}$. We have

$$
B_{n}=\frac{1}{2 \pi i} \int_{|z|=\rho} \frac{\left|H_{n}(z)\right|}{z^{n}} \frac{d z}{z}
$$

so that $\lim _{n \rightarrow \infty} \frac{1}{n} \log \left|r_{n}\right|=\tau^{(0)}+K$ and $\lim _{n \rightarrow \infty} \frac{1}{n} \log \left|\varepsilon_{n}^{(i)}\right|=K-\tau^{(i)}$.

If $H_{n}$ satisfies the condition of the theorem, i.e., if the $\tau^{(i)}$ are all strictly positive and pairwise distinct, and if $\tau=\min _{1 \leq i \leq m-1}\left(\tau^{(i)}\right)>\lim _{n \rightarrow \infty} \frac{1}{n} \log D_{n}=K$, then by Lemma 1 there exists, for any $\varepsilon>0$, a positive integer $H_{0}(\varepsilon)$ such that

$$
\left|p+q_{1} \log \left(\frac{a_{2}}{a_{1}}\right)+\cdots+q_{i} \log \left(\frac{a_{i+1}}{a_{i}}\right)+\cdots+q_{m-1} \log \left(\frac{a_{m}}{a_{m-1}}\right)\right| \geq H^{-\mu-\varepsilon}
$$

for any integers $p, q_{i}$ with $H=\max _{1 \leq i \leq s}\left\{\left|q_{i}\right|\right\} \geq H_{0}(\varepsilon)$, where $\mu=-\frac{\tau^{(0)}+K}{K-\tau}$.

\section{Explicit CASES}

For the linear independence measure of logarithms of prime numbers we have the following results. If we take

$$
\begin{aligned}
H_{n}= & (x-15)^{\left[\alpha_{1} n\right]}(x-16)^{\left[\alpha_{2} n\right]}(x-18)^{\left[\alpha_{3} n\right]} \\
& \times(x-20)^{\left[\alpha_{4} n\right]}\left(87 x^{2}-3024 x+25920\right)^{\left[\alpha_{5} n\right]}
\end{aligned}
$$

with $\alpha_{1}=0.417252, \alpha_{2}=0.508248, \alpha_{3}=0.781200, \alpha_{4}=0.577628, \alpha_{5}=0.005094$, we have

Theorem 3. For any $\varepsilon>0$, there exists a positive integer $H_{0}(\varepsilon)$, such that

$$
\left|p+q_{1} \log 2+q_{2} \log 3+q_{3} \log 5\right| \geq H^{-15.27049-\varepsilon}
$$

for all integers $p, q_{1}, q_{2}, q_{3}$ with $H=\max _{1 \leq i \leq 3}\left\{\left|q_{i}\right|\right\} \geq H_{0}(\varepsilon)$, where $H_{0}(\varepsilon)$ is effectively computable.

If we take

$$
H_{n}=(x-35)^{\left[\alpha_{1} n\right]}(x-36)^{\left[\alpha_{2} n\right]}(x-40)^{\left[\alpha_{3} n\right]}(x-42)^{\left[\alpha_{4} n\right]}(x-45)^{\left[\alpha_{5} n\right]}
$$

with $\alpha_{1}=0.253406, \alpha_{2}=0.378746, \alpha_{3}=0.498637, \alpha_{4}=0.746594, \alpha_{5}=0.247956$, we obtain

Theorem 4. For any $\varepsilon>0$, there exists a positive integer $H_{0}(\varepsilon)$, such that

$$
\left|p+q_{1} \log 2+q_{2} \log 3+q_{3} \log 5+q_{4} \log 7\right| \geq H^{-256.865-\varepsilon}
$$

for all integers $p, q_{1}, q_{2}, q_{3}, q_{4}$ with $H=\max _{1 \leq i \leq 4}\left\{\left|q_{i}\right|\right\} \geq H_{0}(\varepsilon)$, where $H_{0}(\varepsilon)$ is effectively computable.

For the linear independence measure of logarithms of rational numbers, we find the following improvements on earlier results.

Theorem 5. Let $a \geq 4$ be an integer. For any $\varepsilon>0$, there exists a positive integer $H_{0}(\varepsilon)$ such that

$$
\left|p+q_{1} \log \left(1-\frac{1}{a}\right)+q_{2} \log \left(1+\frac{1}{a}\right)\right| \geq H^{-\mu_{1}-\varepsilon}
$$

for all integers $p, q_{1}, q_{2}$ with $H=\max \left\{\left|q_{1}\right|,\left|q_{2}\right|\right\} \geq H_{0}(\varepsilon)$, and a linear independence measure $\mu_{1}$ is given by

$$
\mu_{1}=-\frac{K+\log \left|H_{n}^{1 / n}\left(\xi_{1}\right) x^{-1}\right|}{K+\log \left|H_{n}^{1 / n}\left(\xi_{2}\right) x^{-1}\right|}
$$


where $H_{n}(x)=(x-(a-1))^{n}(x-a)^{n}(x-(a+1)) n, K=\lim _{n \rightarrow \infty} \frac{1}{n} \log D_{n}=2$ with $D_{n}=\operatorname{gcd}[1,2, \ldots, 2 n]$, and $\xi_{1} \notin(a-1, a+1), \xi_{2}, \xi_{3} \in(a-1, a+1)$ are the zeros of the derivative of $H_{n}^{1 / n}(x)$ with $\left|H_{n}\left(\xi_{2}\right)\right|>\left|H_{n}\left(\xi_{3}\right)\right|$.

We have also the following corollaries.

\section{Corollary 6.}

$$
\left|p+q_{1} \log \frac{3}{4}+q_{2} \log \frac{5}{4}\right| \geq H^{-36.86}
$$

for $H=\max \left\{\left|q_{1}\right|,\left|q_{2}\right|\right\} \geq H_{0}(\varepsilon)$.

So we replace the measure $(\mu<88)$ given by Rhin and Toffin $[\mathrm{RT}]$ with $\mu \leq 36.86$. If we use the method that we present in Section 4, we can improve this last measure. We have

Corollary 7. For $H \geq H_{0}$

$$
\left|p+q_{1} \log \frac{3}{4}+q_{2} \log \frac{5}{4}\right| \geq H^{-20.515-\varepsilon}
$$

with $H_{n}=(x-3)^{\left[\alpha_{1} n\right]}(x-4)^{\left[\alpha_{2} n\right]}(x-5)^{\left[\alpha_{3} n\right]}(3 x-10)^{\left[\alpha_{4} n\right]}\left(5 x^{2}-40 x+78\right)^{\left[\alpha_{5} n\right]}$ and $\alpha_{1}=0.99958, \alpha_{2}=0.96100, \alpha_{3}=0.92241, \alpha_{4}=0.07721, \alpha_{5}=0.00146$.

We can also find the linear independence measure of 1 and the three logarithms

$$
\log \left(1-\frac{1}{a}\right), \quad \log \left(1+\frac{1}{a}\right), \quad \log \left(1+\frac{2}{a}\right)
$$

with $a \geq 21$ by using the functions $H_{n}=((x-(a-1))(x-a)(x-(a+1))(x-(a+2)))^{n}$. Moreover, if we use the method of semi-infinite linear programming, we can find the linear independence measures of three logarithms for some $a<21$. For instance, by taking $H_{n}=(x-(a-1))^{[0.7 n]}(x-a)^{n}(x-(a+1))^{n}(x-(a+2))^{[0.6 n]}$, for $a=10$, we have for $H=\max _{i}\left|q_{i}\right| \geq H_{0}$

$$
\left|p+q_{1} \log \left(\frac{9}{10}\right)+q_{2} \log \left(\frac{11}{10}\right)+q_{3} \log \left(\frac{12}{10}\right)\right| \geq H^{-3370.308}
$$

If we take $H_{n}=(x-(a-1))^{[0.958 n]}(x-a)^{[0.802 n]}(x-(a+1))^{n}(x-(a+2))^{[0.791 n]} Q$, where $Q=\left(2 * 5^{42}\right)^{n / 1000}$ for $a=16$, we have for $H=\max _{i}\left|q_{i}\right| \geq H_{0}$,

$$
\left|p+q_{1} \log \left(\frac{15}{16}\right)+q_{2} \log \left(\frac{17}{16}\right)+q_{3} \log \left(\frac{18}{16}\right)\right| \geq H^{-67.251} .
$$

\section{Method of Computation of polynomials} WITH SMALL DEGREE AND MINIMAL NORM

To find the linear independence measure of logarithms of rational numbers, it is enough to find the polynomials related to these numbers which satisfy the conditions of Theorem 2 on a certain interval, i.e., to find the polynomials $H_{n}(x)$ such that

$$
-\lim _{n \rightarrow \infty} \frac{1}{n}\left(\log D_{n}+\log \left(\max _{a \leq x \leq b}\left|H_{n}(x) x^{-n}\right|\right)\right)>0 .
$$

Using the notation (1.5) of the integer transfinite diameter of a real interval with the function $f$ and the ideal $\Delta$, we have

$$
-\lim _{n \rightarrow \infty} \frac{1}{n} \log D_{n}-\log \left(t_{\mathbb{Z},(f, \Delta)}([a, b])\right)>0
$$


with $f=1 / x$ and $\Delta, a, b$ rationals depending on $a_{1}, a_{2}, \ldots, a_{n}$ of Theorem 2 We will give below a method to compute these polynomials. We first present the generalized Müntz-Legendre polynomials which we will use in the process.

4.1. The generalized Müntz-Legendre polynomials. We consider, for $0<$ $a<b$, the scalar product of the real continuous functions on the interval $[a, b]$ defined by

$$
(f, g)_{\varphi}=\int_{a}^{b} f(x) g(x) \varphi(x) d x
$$

where $\varphi$ is a real positive function over the interval $[a, b]$. We put

$$
\|f\|_{2,[a, b]}^{2}=(f, f)_{\varphi} \quad \text { and } \quad\|f\|_{1,[a, b]}=\max _{a \leq x \leq b}(|f(x)| \sqrt{\varphi(x)}) .
$$

By the Gram-Schmidt orthogonalisation process we have

Proposition 8. Let $(v,()$,$) be a real vector space provided with a scalar product$ $(,$,$) and let W$ be a subspace of finite dimension $n+1$ of $V$. Let $w_{0}, w_{1}, \ldots, w_{n}$ be an ordered basis of $W$. Then there exists an ordered basis $L_{0}, L_{1}, \ldots, L_{n}$ of $W$ such that

(1) the subspace generated by $L_{0}, L_{1}, \ldots, L_{i}$ is equal to the subspace generated by $w_{0}, w, \ldots, w_{i}$ for $0 \leq i \leq n$;

(2) the vectors $L_{0}, L_{1}, \ldots, L_{n}$ are orthogonal each other.

Let $F$ be a fixed nonzero polynomial of $\mathbb{R}[x]$ and let $\lambda_{0}, \lambda_{1}, \ldots, \lambda_{n}$ be distinct positive real numbers. We consider $L_{0}, L_{1}, \ldots, L_{n}$ to be the sequence of orthogonal polynomials related to the scalar product $(,)_{\varphi}$ built by the Gram-Schmidt process from the polynomials $p_{0}=F x^{\lambda_{0}}, p_{1}=F x^{\lambda_{1}}, \ldots, p_{n}=F x^{\lambda_{n}}$ such that $L_{i}=\sum_{j<i} C_{i j} p_{j}+p_{i}$ and the $L_{i}$ are an orthogonal basis.

Let $p$ be a generalized Müntz-Legendre polynomial given by $p=\sum_{i=0}^{n} a_{i} F x^{\lambda_{i}}$. When $F=1$, we have the usual Müntz-Legendre polynomial. Suppose here that $a_{n} \neq 0$. Then $p$ can be written also as $p=\sum_{i=0}^{n} b_{i} L_{i}$ with $b_{n}=a_{n}$ and

$$
\|p\|_{2,[a, b]}^{2}=\sum_{i=0}^{n} b_{i}^{2}\left\|L_{i}\right\|_{2,[a, b]}^{2}
$$

so that then

We also have

$$
\|p\|_{2,[a, b]}^{2} \geq b_{n}^{2}\left\|L_{n}\right\|_{2,[a, b]}^{2}=a_{n}^{2}\left\|L_{n}\right\|_{2,[a, b]}^{2} .
$$

$$
\|p\|_{2,[a, b]} \leq \sqrt{b-a}\|p\|_{1,[a, b]},
$$

and then we obtain the inequality

$$
\left|a_{n}\right| \leq \frac{\sqrt{b-a}}{\left\|L_{n}\right\|_{2,[a, b]}}\|p\|_{1,[a, b]} .
$$

4.2. Computation of polynomials with small degree and minimal norm. In this paragraph, we denote by $p_{n}$ a polynomial in $\mathbb{Z}[x]$ with degree $n$ such that $\left\|p_{n}\right\|_{1,[a, b]}$ is minimal with $\varphi(x)=x^{-d}, d \in \mathbb{N}^{*}$. We give an algorithm which allows us to find the polynomials with minimal norm on a real interval by using generalized Müntz-Legendre polynomials, the LLL algorithm, and a method adapted from the simplex method. We use the same outline as Habsieger and Salvy [HS] but different techniques in detail, i.e., 
1. find a good upper bound for $\left\|p_{n}\right\|_{1,[a, b]}$;

2. use this bound to deduce polynomials that are necessarily factors of $p_{n}$;

3. perform an exhaustive search for the missing factors.

The process in detail is as follows:

a) Good upper bound for $\left\|p_{n}\right\|_{1,[a, b]}$. For $n$ fixed, if we have the minimal polynomials with degree $m$ and $m<n$, then we can take the first good bound

$$
c_{n}=\min _{m}\left\|p_{m} p_{n-m}\right\|_{1,[a, b]} .
$$

As remarked in [HS], the natural basis of the polynomials is not the best, so we replace it by a basis obtained by the LLL algorithm. Let $H$ be a lattice with basis $\left(e_{i}\right)_{1 \leq i \leq m}$. Then the LLL algorithm furnishes a new LLL-reduced basis $\left(f_{i}\right)_{1 \leq i \leq m}$ of $H$, i.e., such that the norm (euclidean norm) of vectors $f_{i}$ is small, and in particular such that the vector $f_{1}$ is not so far from being the smallest nonzero vector in $H$. More explicitly, on the interval $[a, b]$, suppose that the minimal polynomial $p_{n}$ with degree $n$ has the factor $F$ with $\operatorname{deg} F=k$. Then $p_{n}=F Q$, where $Q=$ $\alpha_{0}+\alpha_{1} x+\cdots+\alpha_{k} x^{n-k}$ and the $\alpha_{i}$ are integers. We consider the lattice $H$ generated by the $n-k+1$ vectors $\left(F\left(x_{i}\right)\right)_{1 \leq i \leq N},\left(F\left(x_{i}\right) x_{i}\right)_{1 \leq i \leq N}, \ldots,\left(F\left(x_{i}\right) x_{i}^{n-k}\right)_{1 \leq i \leq N}$. We apply the LLL algorithm to these $n-k+1$ vectors and obtain a polynomial $F Q$ which is in general a polynomial that provides a good upper bound. We observe in our calculations that this method often produces the minimal polynomial $p_{n}$.

b) Search of the necessity factors of $p_{n}$ by using this bound. In our process, we start by using the generalized Müntz-Legendre method with this bound to give an upper bound for the exponents of the polynomials in $\mathbb{Z}[x]$ which have a root at the endpoints of the interval $[a, b]$. We compute explicitly the Gram matrix with the basis $F,\left(a_{i} x-b_{i}\right) F, \ldots,\left(a_{i} x-b_{i}\right)^{n-\operatorname{deg} F} F$, where $\frac{b_{i}}{a_{i}} \in[a, b]$ and $F$ is of the form $(x-a)^{k_{1}}(x-b)^{k_{2}} \prod_{i}\left(a_{i} x-b_{i}\right)^{k_{i+2}}$. If $p_{n}=\alpha_{0} F+\alpha_{1}\left(a_{i} x-b_{i}\right) F+\cdots+\alpha_{n-\operatorname{deg} F}\left(a_{i} x-\right.$ $\left.b_{i}\right)^{n-\operatorname{deg} F} F$, we compute the Gram matrix with the scalar product $(,)_{\varphi}$ and we use the Gram-Schmidt process on this matrix. The last diagonal term obtained gives $\left\|L_{n}\right\|_{2,[a, b]}^{2}$. Thus, we can maximize $\left|\alpha_{0}\right| \leq c_{n} \sqrt{b-a} /\left(\left\|L_{n}\right\|_{2,[a, b]}\right)$, by (4.4), and so that $p_{n}$ has a zero of order $>k_{i+2}$ at the point $b_{i} / a_{i}$. We repeat this until we do not find any more new factors of the first degree. We do not consider here the case of the factors of degree 2 or more for which it would be necessary to use resultants.

c) Exhaustive search. We solve the system of linear inequalities that we obtain by taking particular values of the variable and thus find the missing factors of polynomial of minimal norm. After having found the necessity factors, $p_{n}$ is written in the form $p_{n}=F Q(x)$, where $Q(x)$ is a polynomial of degree $k=n-\operatorname{deg} F$ whose unknown coefficients are to be determined. We consider the system of linear inequalities with $k+1$ variables:

$$
\left|F\left(x_{i}\right) Q\left(x_{i}\right)\right| \leq c_{n} \quad \text { for } 1 \leq I \leq m,
$$

where $x_{i}$ are control points which are different from the roots of $F(x), m \geq k+1$ is the number of such points and $Q(x)=\alpha_{0}+\alpha_{1} x+\cdots+\alpha_{k} x^{k}$. The coefficients of $Q$ are thus the unknown variables of the system (4.5). This system defines a polyhedron of which we must determine the integer points. We solve this system of inequalities with a method adapted from the simplex method and the LLL algorithm. We thus have several polynomials (there are perhaps some polynomials $q$ such that $\|Q\|_{1,[a, b]} \geq c_{n}$ because of the choice of the control points and the 
numerical inaccuracies in the pivoting procedure). We choose the best polynomial for $Q$, i.e., one of those for which the standard norm $\|*\|_{1,[a, b]}$ is smallest. Thus, one obtains a polynomial $p_{n}$ which is appropriate. All calculations were carried out with Pari software.

\section{Study of THE LineAR InDEPENDENCE MEASURE}

After having the minimum polynomials of degree $n$ fixed, we will seek the best combination of the exponents of factors of the polynomials to compute the measure. To apply linear programming, we limit either the numerator or the denominator of the function of the measure (2.3). We can thus find a linear function of the exponents of the factors by using the logarithm of the function.

We solve a problem of the following type. Find

$$
\max _{C} \min _{x \in X} g(x, C)
$$

where $g(x, C)$ is a linear function of $C=\left(c_{1}, \ldots, c_{k}\right)$ and $X$ is a compact subset of $\mathbb{C}$, the maximum being taken for $c_{i} \geq 0$ for $i=1, \ldots, k$.

A traditional solution consists in choosing very many control points $\left(x_{j}\right)_{1 \leq j \leq N}$ and to solve the linear programming problem

$$
\max _{C} \min _{1 \leq j \leq N} g\left(x_{i}, C\right) .
$$

However, the result obtained depends on the particular control points selected.

The idea of semi-infinite linear programming [AN] consists in repeating the preceding process while adding new control points each time and checking that this process converges to $m$, the value of the linear form for a choice of $C$ optimum, according to the following algorithm.

(1) One chooses an initial value of $C$ be $C^{(0)}$ and we calculate

$$
m_{0}^{\prime}=\min _{x \in X} g\left(x, C^{(0)}\right) .
$$

(2) One chooses a set of control points $X^{(0)}$ in $X$. It is clear that we have $m_{0}^{\prime} \leq m \leq m_{0}$, where $m_{0}=\min _{x \in X^{(0)}} g\left(x, C^{(0)}\right)$.

(3) We add to $X^{(0)}$ the points where $g\left(x, C^{(0)}\right)$ admits local minima and we obtain a set $X^{(1)}$ of control points.

(4) We solve the problem of linear programming $\max _{C} \min _{x_{j} \in X^{(1)}} g\left(x_{j}, C\right)$. We then obtain a new value of $C$ be $C^{(1)}$ and a result of the linear programming equal to $m_{1}^{\prime}=\min _{x \in X} g\left(x, C^{(1)}\right)$. We then have $m_{0}^{\prime} \leq m_{1}^{\prime} \leq m \leq m_{1} \leq m_{0}$, where $m_{1}=\min _{x \in X^{(1)}} g\left(x, C^{(1)}\right)$.

(5) We repeat operations (2) to (4) and, thus, we find two sequences $\left(m_{i}\right)$ and $\left(m_{i}^{\prime}\right)$ such that $m_{0}^{\prime} \leq m_{1}^{\prime} \leq \cdots \leq m_{i}^{\prime} \leq m \leq m_{i} \leq \cdots \leq m_{1} \leq m_{0}$.

We stop as soon as there is a good approximation, for example, $m_{i}-m_{i}^{\prime}<10^{-6}$. Supposing that $p$ iterations are enough, we then take $m_{p}$ as an approximation of $m$.

In $\mathrm{AN}$ we can find the general theory of semi-infinite linear programming. All computations are carried out in PASCAL.

Thus, in theory, we find a method to seek the function which makes it possible to compute a linear independence measure of the logarithms of rational numbers. We search initially for the best combination of polynomials such that the polynomials are in the ideal $(\Delta, x)$ where $\Delta$ is generated by the rational numbers used by using the generalized Müntz-Legendre polynomials, the LLL algorithm, and a method 
adapted from the simplex method. Then we search for the best exponents of the polynomials by semi-infinite linear programming, such that the absolute value of $F(x)$ generated by these polynomials is the smallest possible in the considered interval. If we obtain a function $F(x)$, which satisfies the conditions (4.1), then we find a linear independence measure of the logarithms of rational numbers or prime numbers associated to this function.

By using this method, we find the functions for 3.1 and (3.2). We also prove that the polynomials $Q_{4}, Q_{5}$ and $Q_{6}$ in (1.4) are obtained as factors of polynomials $P$ in $I_{n, \Delta}$ having a small degree and a minimal norm. By using this method, we find the same exponents of the polynomials and same measure. If we continue to improve the numerator of the function of the measure, we find that the measure $\mu(1, \log 2, \log 3) \leq 7.6155$ (replacing 7.616 ) with the exponents $0.55255,0.70451$, $0.44744,0.10884,0.03842$ and 0.05489 replaces $0.552418,0.704324,0.447582$, $0.109072,0.038934$, and 0.054368 , respectively.

\section{REFERENCES}

[AM1] F. Amoroso, Sur le diamètre transfini entier d'un intervalle réel, Ann. Inst. Fourier (Grenoble), 40 (1990), 885-911. MR 92j:11070

[AM2] F. Amoroso, $f$-Transfinite diameter and number-theoretic applications, Ann. Inst. Fourier (Grenoble) 43 (1993), 1179-1198. MR 95d:11091

[AN] E. J. Anderson and P. Nash, Linear programming in infinite-dimensional spaces, WileyInterscience Publication (1987). MR 88f:90180

[BW] A. Baker ad G. Wüstholz, Logarithmic forms and group varieties, J. Reine Angew. Math. 442 (1993), 19-62. MR 94i:11050

[BE] P. Borwein and T. Erdelyi, The integer Chebyshev problem, Math. Comp. 65 (1996), 661681. MR 96g:11077

[DA] V. Danilov, Rational approximations of some functions at rational points, Mat. Zametki 24 (1978), 449-458, 459. MR 80a:10046

[FRS] V. Flammang, G. Rhin and C. J. Smyth, The integer transfinite diameter of intervals and totally real algebraic integers, J. Théor. Nombres Bordeaux 9 (1997), 137-168. MR 98g:11119

[HA] M. Hata, Rational approximations to $\pi$ and some other numbers, Acta Arith. 63 (1993), 335-349. MR 94e:11082

[HS] L. Habsieger and B. Salvy, On integer Chebyshev polynomials, Math. Comp. 66 (1997), 763-770. MR 97f:11053

[LLL] A. K. Lenstra, H. W. Lenstra and L. Lovasz, Factoring polynomials with rational coeffcients, Math. Ann. 261 (1982), 515-534. MR 84a:12002

[NI] E. M. Nikisin, Irrationality of the values of functions $F(x, s)$, Mat. Sb. 109 (1979), 410417. MR 82b:10044a

[RH] G. Rhin, Approximants de Padé et mesures effectives d'irrationalité, Séminaire de Théorie des Nombres, Paris 1985/1986, Prog. Math. 71 (1987), 155-164. MR 90k:11089

[RT] G. Rhin and P. Toffin, Approximants de Padé simultanés de logarithmes, J. Number Theory 24 (1986), 284-297. MR 88i:41033

[RU] E. A. Rukhadze, A lower bound for the approximation of $\ln 2$ by rational numbers, Vestnik Moskov. Univ. Ser. I Mat. Mekh. 1987, no. 6, 25-29, 97. MR 89b:11064

[VI] C. Viola, On Siegel's method in Diophantine approximation to transcendental numbers, Rend. Sem. Mat. Univ. Politec. Torino 53 (1995), 455-469. MR 98f:11076

[WA] M. Waldschmidt, Minorations de combinaisons linéaires de logarithmes de nombres algébriques, Can. J. Math. 45 No. 1, (1993), 176-224. MR 94f:11065

Département de Mathématique, Université de Metz, Ile du Saulcy, 57045 Metz Cedex 1, France

E-mail address: wu@poncelet.univ-metz.fr 